\title{
TRANSLUCENCIA NUCAL AUMENTADA Y CARIOTIPO NORMAL
}

\author{
Tamara Illescas M. ${ }^{1}$, Javier Pérez P. ${ }^{1}$, Pilar Martínez T. ${ }^{1}$, Belén Santacruz M. ${ }^{1}$, \\ Begoña Adiego B. ${ }^{1}$, Esther Barrón A. ${ }^{1}$ \\ 1 Delta Ecografía, Madrid, España.
}

\section{RESUMEN}

Antecedentes: La exploración ecográfica entre las semanas 11 y 14 tiene un papel fundamental en el cribado de anomalías cromosómicas, siendo la medida de la translucencia nucal un método bien establecido y ampliamente aceptado para este fin. Objetivo: Evaluar retrospectivamente la evolución de los fetos con translucencia nucal aumentada y cariotipo normal. Método: Se recogieron los datos de 104 fetos con TN $\geq$ percentil 95 (p95) entre las semanas 11 y 14, evaluados en nuestro centro. En los 61 que resultaron euploides, se estudió la incidencia de anomalías estructurales diagnosticadas tanto prenatalmente como tras el nacimiento, así como las pérdidas fetales anteparto. De estos fetos, nacieron sanos el $80 \%$ cuando la TN estaba entre p95 y $3,4 \mathrm{~mm}$, el $50 \%$ con TN entre 3,5 y $4,4 \mathrm{~mm}$, el $30 \%$ con TN entre 4,5 y $5,4 \mathrm{~mm}$, y el $18 \%$ con TN $\geq 5,5 \mathrm{~mm}$. Entre los 4 recién nacidos euploides con patología, hubo 3 con cardiopatía. Conclusión: La presencia de TN aumentada entre las 11-14 semanas en fetos euploides se asocia a un incremento del riesgo de anomalías estructurales mayores, principalmente cardiacas. La prevalencia de malformaciones aumenta significativamente con $\mathrm{TN}>3,5 \mathrm{~mm}$, y el pronóstico gestacional adverso empeora conforme aumenta la medida de la TN.

\section{PALABRAS CLAVE: Translucencia nucal, malformación fetal, cariotipo normal}

\section{SUMMARY}

Background: Ultrasound scan has a main role at the 11-14 weeks screening for chromosomal abnormalities. The measurement of nuchal translucency (NT) thickness is a widespread stablished method to achieve this target. Objective: To assess retrospectively the outcome of fetuses with increased nuchal translucency and normal karyotype. Method: Data were collected from 104 fetuses with NT > 95th percentile at 11 to 14 weeks of gestation, followed in our institution. The sixty one euploid fetuses were studied in order to determine the incidence of structural abnormalities, diagnosed either before or after delivery, as well as antenatal fetal loss. Among these fetuses, there were no malformations at birth in $80 \%$ for NT between the 95th percentile and $3.4 \mathrm{~mm} ; 50 \%$ for NT between 3.5 and $4.4 \mathrm{~mm} ; 30 \%$ for NT of $4.5-5.4 \mathrm{~mm}$; and $18 \%$ for NT $\geq 5.5 \mathrm{~mm}$. There were 4 euploid newborns with some kind of structural defect at birth, 3 of them presented a cardiac malformation. Conclusion: The presence of increased NT at 11-14 weeks scan in euploid fetuses it's associated with a higher risk of major structural abnormalities, mainly cardiac ones. The prevalence of malformations is significantly increased for NT $>3.5 \mathrm{~mm}$, and the adverse perinatal outcome is directly associated with the thickness of NT.

\section{KEY WORDS: Nuchal translucency, fetal malformation, normal karyotype}




\section{INTRODUCCIÓN}

La medida de la translucencia nucal se ha revelado como una herramienta de trabajo imprescindible en el diagnóstico prenatal. Su determinación por ecografía entre las semanas 11 y 14 de gestación, combinada con la edad materna y los marcadores bioquímicos $B \mathrm{HCG}$ (fracción $B$ de la gonadotropina coriónica humana) y PAPP-A (proteína A asociada a la gestación), ha demostrado ser de gran utilidad para el cribado de cromosomopatías en el primer trimestre (1). Sin embargo, se ha observado también una relación entre el aumento de la TN y una amplia serie de malformaciones (entre los que destacan los defectos cardíacos mayores), así como diversos síndromes genéticos $(2,3)$.

El objetivo del presente estudio ha sido la evaluación retrospectiva de la evolución de estos fetos euploides con TN aumentada. Hemos querido demostrar la necesidad de un control más exhaustivo en estos fetos, que incluya un estudio ecocardiográfico, dada la mayor incidencia de resultados adversos a pesar del cariotipo normal.

\section{MATERIAL Y MÉTODO}

Entre febrero de 2004 y agosto de 2008, en el centro Delta Ecografía, se examinó mediante ultrasonidos a 104 fetos con $\mathrm{TN} \geq$ p95 en relación a su longitud cráneo-caudal ( $\mathrm{CRL})$, obtenida entre las semanas 11 y 14, siguiendo los criterios de la Fetal Medicine Foundation (http://www.fetalmedicine. com/fmf/online-education/01-11-136-week-scan/). Se determinó en todos ellos el cariotipo, mediante técnicas invasivas (amniocentesis o biopsia corial). En 61 fetos euploides, se estudió las pérdidas anteparto y la incidencia de anomalías estructurales, diagnosticadas tanto prenatalmente como en el estudio posmortem o tras el nacimiento. Se realizó un seguimiento ecográfico periódico, y se revisaron los resultados a través de los datos obtenidos de los registros ecográficos, así como mediante entrevista personal o telefónica con las propias pacientes, tras el parto o la interrupción legal del embarazo (ILE).

\section{RESULTADOS}

De los 104 fetos con TN $\geq$ p95, 61 tuvieron cariotipo normal $(58,7 \%)$. En fetos euploides el valor medio de CRL al diagnóstico de TN aumentada fue de $59 \mathrm{~mm}$ y la edad materna media fue 32 años (rango: 25-39 años) y 36 años (rango: 28-43 años) en aneuploides.

Se agrupó a los fetos según el valor de TN, en cuatro grupos: TN entre el percentil 95 y $3,4 \mathrm{~mm}$ (primer grupo), de 3,5 a 4,4 mm (segundo grupo), de 4,5 a 5,4 $\mathrm{mm}$ (tercer grupo) y con $\mathrm{TN} \geq 5,5 \mathrm{~mm}$ (cuarto grupo) (Tabla I).

Tabla I

\section{RESULTADOS SEGÚN MEDIDA DE LA TN, EN 104 FETOS CON TN $\geq$ P95}

\begin{tabular}{lrrrrr}
\hline \multicolumn{3}{c}{ Aneuploides } & & \multicolumn{3}{c}{ Euploides } \\
TN & $\mathrm{n}(\%)$ & ILE & Abortos & RNP & RNS \\
$\mathrm{nm})$ & & $\mathrm{n}(\%)$ & $\mathrm{n}(\%)$ & $\mathrm{n}(\%)$ & $\mathrm{n}(\%)$ \\
\hline $\mathrm{P} 95-3,4$ & $4(16)$ & 0 & 0 & $1(4)$ & $20(80)$ \\
$3,5-4,4$ & $11(39,5)$ & $2(7)$ & $1(3,5)$ & 0 & $14(50)$ \\
$4,5-5,4$ & $6(47,5)$ & $2(15)$ & $1(7,5)$ & 0 & $4(30)$ \\
$\geq 5,5$ & $22(58)$ & $3(8)$ & $3(8)$ & $3(8)$ & $7(18)$ \\
\hline
\end{tabular}

TN: translucencia nucal. P: percentil. ILE: interrupción legal del embarazo. RNP: recién nacido patológico. RNS: recién nacido sano.

En el primer grupo, se hizo amniocentesis en el $48 \%$ de los casos; el porcentaje de amniocentesis frente a biopsia corial desciende progresivamente, y en el segundo grupo es de $32 \%$; los dos últimos grupos están prácticamente a la par, con $15 \%$ y $16 \%$ de amniocentesis, respectivamente (Tabla II).

Tabla II

\section{AMNIOCENTESIS Y BIOPSIAS CORIALES SEGÚN MEDIDA DE LA TN, EN 104 FETOS CON $\mathrm{TN} \geq \mathrm{P} 95$}

\begin{tabular}{lcccccc}
\hline $\begin{array}{c}\text { TN } \\
(\mathrm{mm})\end{array}$ & $\begin{array}{c}\text { AMN } \\
(\%)\end{array}$ & $\begin{array}{c}\text { BC } \\
(\%)\end{array}$ & \multicolumn{2}{c}{ Aneuploides } & \multicolumn{2}{c}{ Euploides } \\
\hline P 95-3,4 & 48 & 52 & 1 & 3 & 11 & 10 \\
$3,5-4,4$ & 32 & 68 & 4 & 7 & 5 & 12 \\
$4,5-5,4$ & 15 & 85 & 0 & 6 & 2 & 5 \\
$\geq 5,5$ & 16 & 84 & 2 & 20 & 4 & 12 \\
\hline
\end{tabular}

TN: translucencia nucal. AMN: amniocentesis. BC: biopsia corial. $\mathrm{P}$ : percentil.

En el primer grupo, el cariotipo fue anómalo en el $16 \%$ de los casos, y se obtuvo un $80 \%$ de nacidos sanos. En el segundo grupo, el porcentaje de nacidos sanos fue del $50 \%$, y las aneuploidías alcanzaron el $39,5 \%$. En el tercer grupo hubo $47,5 \%$ de cariotipo alterados y un $30 \%$ de gestaciones llevadas a término con normalidad. En el último grupo, los nacidos sanos fueron el $18 \%$, los patológicos el $8 \%$, el $58 \%$ fueron aneuploides y hubo $16 \%$ de pérdidas (aborto espontáneo o ILE) (Tabla I).

Entre los fetos euploides detallamos a continuación 
los resultados patológicos:

1. Casos de recién nacidos patológicos. Todos habían presentado $\mathrm{TN} \geq 5,5 \mathrm{~mm}$, excepto el caso 1 , que había tenido una $\mathrm{TN}<3,5 \mathrm{~mm}$.

Caso 1: Braquicefalia, bajo peso al nacer $(2.100 \mathrm{~g}$ a las 39 semanas de edad gestacional) y pies equinovaros.

Caso 2: Comunicación interventricular (CIV), coartación de aorta, retraso del crecimiento intrauterino (CIR). Se trataba de una gestación gemelar bicorial biamniótica, que cursó con rotura prematura de membranas en la semana 31 , y terminó con cesárea programada en la semana 35; el gemelo patológico pesó $1.540 \mathrm{~g}$, y el otro $1.800 \mathrm{~g}$.

Caso 3: Cardiopatía severa y síndrome de Noonan; falleció a los 3 meses y medio de vida. Se trataba de una gestación gemelar bicorial biamniótica que finalizó por parto eutócico en la semana 35 (con un peso neonatal de $2.400 \mathrm{~g}$ ).

Caso 4: Agenesia de ductus venoso (Figura 1), comunicación interauricular (CIA), comunicaciones interventriculares múltiples pequeñas, persistencia de ductus arterioso, estenosis pulmonar, paladar ojival, edema nucal, sospecha de síndrome de Noonan.

2. Casos de interrupción legal del embarazo.

Caso 1: Lisencefalia, malposición de manos y pies, estómago y orejas hipoplásicos, CIR.

Caso 2: Ventriculomegalia cerebral (Figura 2), hernia diafragmática (Figura 3).

Caso 3: Atresia tricuspídea (Figura 4).

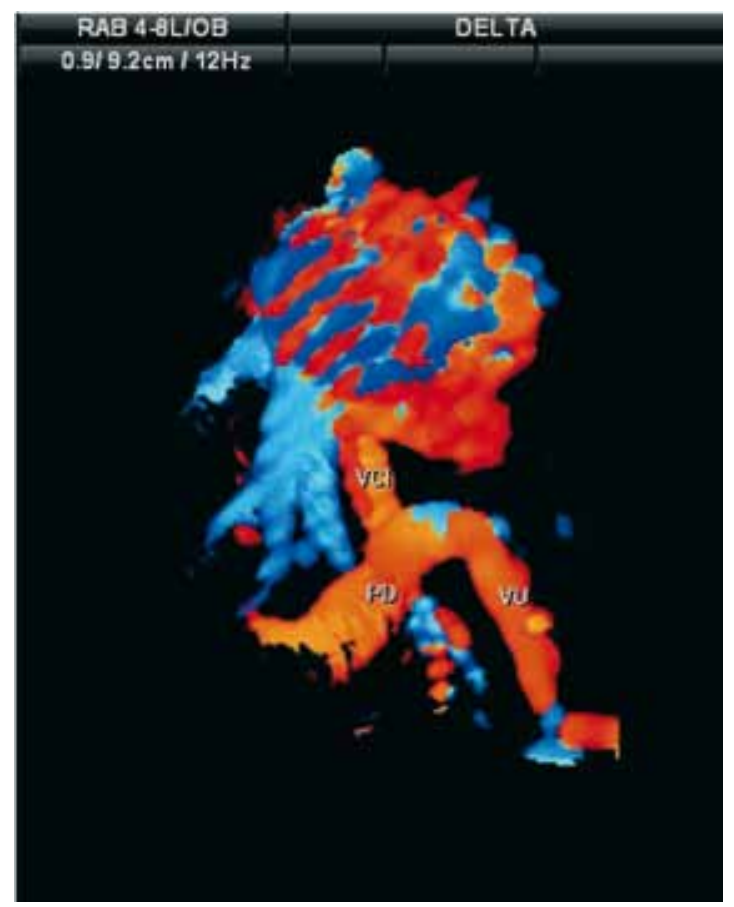

Figura 1. Agenesia de ductus venoso.

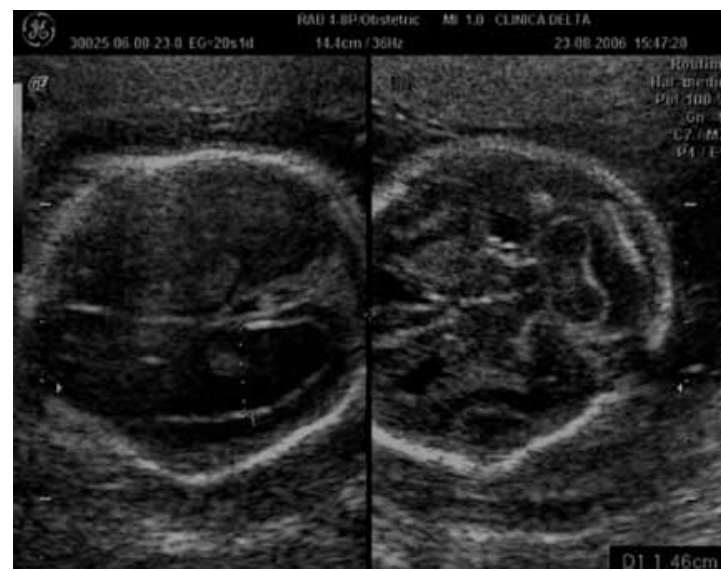

Figura 2. Ventriculomegalia cerebral.

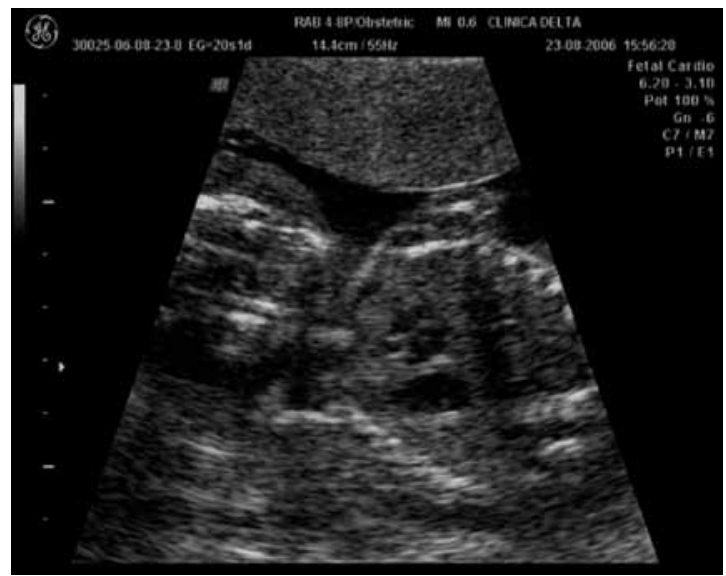

Figura 3. Hernia diafragmática.

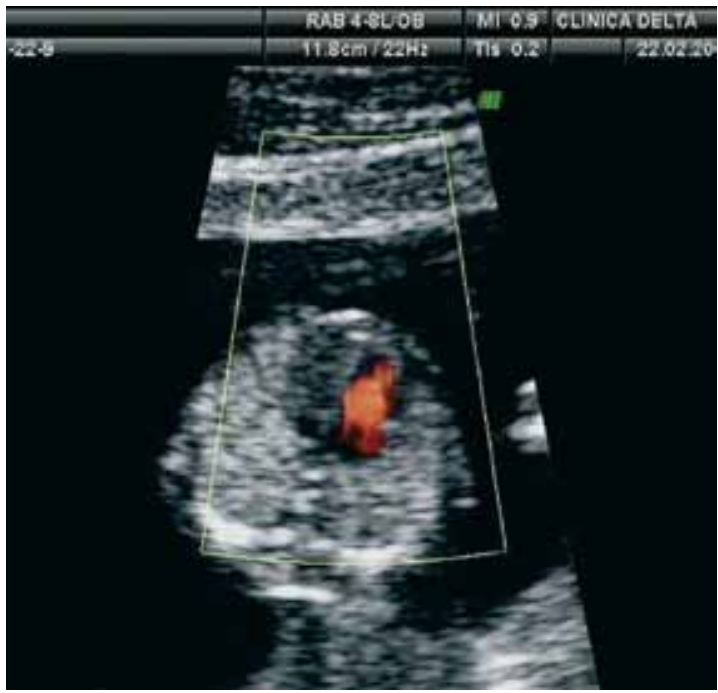

Figura 4. Atresia tricuspídea. 
Casos 4-7: Higroma/edema (Figura 5).

3. Caso de muerte fetal. Se constata en un caso muerte fetal a las 33 semanas. La gestación había cursado con higroma quístico, edema, derrame pleural y arteria umbilical única. El hidrotórax y el edema cutáneo habían desaparecido en la semana 19.

4. Casos con nacidos vivos con bajo peso, no CIR. Uno de ellos fue una gestación simple, de la que resultó un neonato de $2.200 \mathrm{~g}$ a las 39 semanas, que tuvo una evolución favorable. El segundo caso nació por cesárea urgente de una gestación gemelar bicorial biamniótica, con un peso de $1.980 \mathrm{~g} \mathrm{a}$ las 36 semanas.

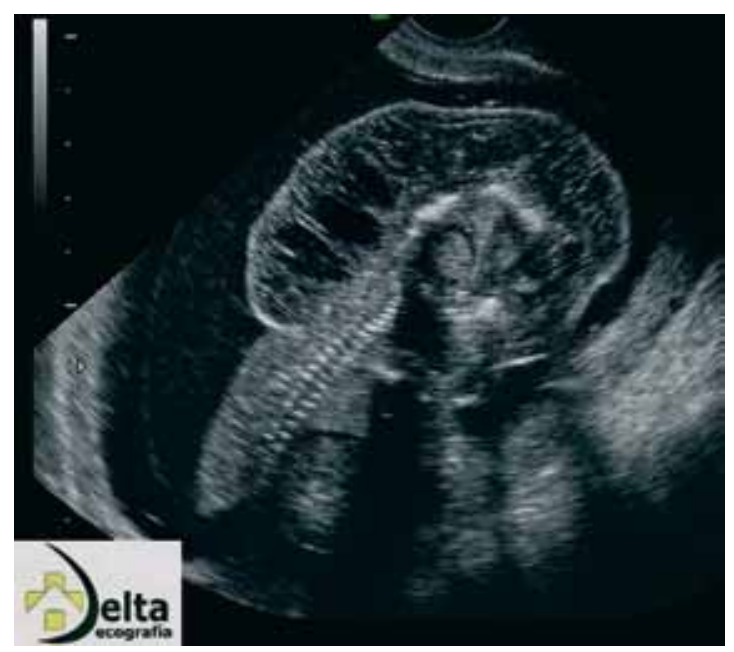

Figura 5. Higroma quístico.

\section{DISCUSIÓN}

La exploración ecográfica entre las semanas 11 y 14 tiene un papel fundamental en el cribado de anomalías cromosómicas. La medida de la translucencia nucal es un método bien establecido y ampliamente aceptado para este fin y se ha ido incorporando progresivamente a la práctica clínica rutinaria. La técnica para su evaluación esta bien descrita y estandarizada (1).

La determinación de la TN en combinación con la edad materna y los marcadores bioquímicos de primer trimestre (PAAP-A y b HCG), puede detectar aproximadamente el $90 \%$ de los fetos con trisomía 21 y otras anomalías cromosómicas mayores (2). La sensibilidad para la detección de síndrome de Down utilizando este marcador de forma aislada puede alcanzar el $60-70 \%$ con una tasa muy baja de falsos positivos. Sin embargo, una TN aumentada puede aportar más información que la rela- cionada con el cariotipo. Múltiples trabajos coinciden en la utilidad de este marcador no sólo para el diagnóstico de aneuploidías, sino también de malformaciones estructurales, síndromes genéticos, displasias, y disrupciones. La medida de TN aporta información pronóstica de carácter general a la gestación, de manera que el grosor se asocia, de forma directamente proporcional, con el pronóstico perinatal adverso (3).

El término translucencia nucal aumentada se refiere a la medida por encima del percentil 95 y se emplea independientemente de que la colección de fluido esté septada (higroma) o no, confinada al cuello o envolviendo al feto por completo. En un trabajo retrospectivo con 223 fetos de 11-14 semanas con TN aumentada, Ducarme y cols (4) observaron una incidencia de aneuplodías del $55 \%$ en aquellos fetos con colección septada (higroma) mientras que la incidencia era solamente del $14,2 \%$ en aquellos con TN aumentada sin higroma. Además, entre aquellos fetos con cariotipo normal, fueron estructuralmente normales sólo el $44 \%$ de los que tuvieron higroma frente al $93 \%$ de los que presentaron TN aumentada no septada.

En nuestros resultados la edad gestacional media al diagnóstico de TN aumentada fue similar en ambos grupos (12 semanas de media). Destaca una mayor edad de las pacientes en el grupo de fetos aneuploides, en comparación con los euploides (de 32 a 36 años de media). Observamos que el porcentaje de biopsias coriales aumenta con la medida de la TN. Esto se explica por la mayor probabilidad de cromosomopatía con valores de TN mayores, lo que obliga a descartarla lo más precozmente posible, para facilitar las decisiones terapéuticas y disminuir la ansiedad de los padres.

Múltiples y diferentes mecanismos se han relacionado con el incremento de TN entre las semanas 11 y 14: disfunción cardiaca (en relación con malformaciones del corazón y los grandes vasos), congestión venosa en cabeza y cuello, alteración en la composición de la matriz extracelular, fallo en el drenaje linfático (originado por un desarrollo anómalo o tardío del sistema linfático), disminución de movimientos fetales, anemia fetal o hipoproteinemia e infección fetal. Todas estas posibles causas son de gran utilidad a la hora de realizar una exploración dirigida, fundamentalmente en aquellas gestaciones euploides evolutivas tras el estudio del cariotipo fetal.

La presencia de TN aumentada en un feto con cariotipo normal significa un riesgo mayor de cardiopatías (5) y otros síndromes (6-8), si bien el pronóstico perinatal no empeora de forma estadísticamente significativa hasta que el grosor de la medida 
alcanza 3,5 mm o más (> p99). La probabilidad de tener un hijo sin anomalías mayores es del $70 \%$ para valores de TN entre 3,5-4,4 mm, 50\% para TN de 4,5-5,4 mm, 30\% para TN de 5,5-6,4 $\mathrm{mm}$ y $15 \%$ para $\mathrm{TN}$ de $6,5 \mathrm{~mm}$ o más (3). Estos datos son muy similares a los obtenidos de nuestra casuística (Tabla I). En nuestro grupo de fetos euploides, los eventos adversos son más frecuentes según aumenta la medida de la TN, sobre todo a partir de $3,5 \mathrm{~mm}$. Los estudios de necropsia de los fetos sometidos a ILE mostraron malformaciones severas y múltiples, así como higroma y edema. De los 4 RN con patología, el $75 \%$ pertenecían al grupo de TN $\geq 5,5 \mathrm{~mm}$.

Si el feto sobrevive hasta la semana 20-22, y una exploración dirigida y detallada de alta resolución no detecta ninguna anomalía, el riesgo de evolución perinatal adversa y retraso del desarrollo postnatal, no está incrementado de manera estadísticamente significativa, según afirman Nicolaides y cols (3). Sin embargo, en nuestro estudio cabe destacar dos nacidos con bajo peso, y una muerte fetal en la semana 33 de gestación, en un feto con hallazgo de edema que había resuelto en la semana 19.

Atzei y cols (9) comunicaron que la prevalencia de malformaciones cardiacas se incrementa exponencialmente con el grosor de la TN. Souka y cols (3), en un trabajo con más de 4.000 fetos con TN aumentada y cariotipo normal, observaron un incremento en el pronóstico perinatal adverso a expensas de una mayor prevalencia de defectos cardiacos severos, hernia diafragmática, onfalocele, body stalk anomaly, secuencia de aquinesia y deformación fetal, displasias esqueléticas raras y síndromes genéticos.

Souka y cols (5) también investigaron las malformaciones fetales mayores detectables en las ecografías regladas del primer y segundo trimestre de gestación. De los fetos patológicos, hallaron un antecedente de TN aumentada en el 35,7\%, así como en el $25 \%$ de los fetos que presentaron una cardiopatía. Por ello, éste y otros autores, recomiendan un examen morfológico precoz, que incluya ecocardiografía, en casos de detección de TN aumentada entre las semanas 11-14.

Si bien nuestra casuística no es lo suficientemente amplia para obtener conclusiones significativas, nosotros también hemos observado una mayor incidencia de resultados adversos conforme aumenta el valor de TN, y de estos, la mayoría consistía en alteraciones cardiacas, y la mitad tenía al menos sospecha de síndrome de Noonan. Esto nos reafirma la recomendación del estudio de estos fetos, por especialistas en diagnóstico prenatal ultrasonográfico, mediante un estudio anatómico precoz exhaustivo, con especial atención a la ecocardiografía.

Para seleccionar los fetos tributarios de pruebas complementarias del estudio anatómico, se ha propuesto el uso de marcadores de segundo orden. Aunque no existe aún un protocolo de actuación ampliamente establecido, sí hay trabajos que han demostrado la utilidad del estudio de otros marcadores ecográficos en fetos con TN aumentada y cariotipo normal. La presencia de flujo diastólico ausente o reverso en el ductus venoso y la presencia de regurgitación tricuspídea se asocian con un incremento en el riesgo relativo de cardiopatías. Su normalidad, por el contrario, disminuye este riesgo. De este modo, su valoración en casos con TN mayor al p95 puede ser de gran ayuda para determinar qué fetos se beneficiarán de un examen morfológico y ecocardiográfico precoz en un centro de referencia.

Se han establecido diferentes protocolos de valoración precoz teniendo en cuenta las limitaciones impuestas tanto por la edad gestacional y el tamaño de las estructuras, como por el desarrollo incompleto de otras, ya que, por ejemplo, el desarrollo del cuerpo calloso y el vérmix cerebeloso no finaliza hasta las 18 y 16 semanas de gestación, respectivamente. Otras anomalías, como las cardiacas, están sujetas a cambios evolutivos que sólo se manifiestan con el progreso del embarazo (por ejemplo, la estenosis mitral y el síndrome de ventrículo izquierdo hipoplásico asociado) por lo que el establecimiento de un pronóstico claro en etapas gestacionales tempranas ofrece una gran dificultad. Sin embargo, algunos autores sostienen que la gran mayoría de las alteraciones fetales asociadas con el aumento de TN se pueden diagnosticar antes de la semana 14 (3).

Queremos destacar que la valoración morfológica precoz de aquellos fetos euploides con TN > p95 ofrece, en manos expertas, un elevado valor predictivo negativo, de gran importancia a la hora de disminuir la ansiedad de los futuros padres.

\section{CONCLUSIÓN}

Dado el evidente aumento del riesgo de eventos adversos en gestaciones con TN aumentada, incluso tras descartar las cromosomopatías, es importante el seguimiento de estos fetos.

\section{BIBLIOGRAFÍA}

1. Nicolaides $\mathrm{KH}$. Nuchal translucency and other first trimester sonographic markers of chromosomal abnormalities. Am J Obstet Gynecol 2004;191:45-67. 
2. Nicolaides $\mathrm{KH}$, Heath $\mathrm{V}$, Cicero S. Increased fetal nuchal translucency at 11-14 weeks. Prenat Diagn 2002;22:308-15.

3. Souka AP, Von Kaisenberg CS, Hyett JA, Sonek JD, Nicolaides $\mathrm{KH}$. Increased nuchal translucency with normal karyotype. Am J Obstet Gynecol 2005;192:100521.

4. Ducarme G, Graesslin O, Alanio E, Bige V, Gaillard D, Gabriel R. Increased nuchal translucency and cystic hygroma in the first trimestre: prenatal diagnosis and neonatal outcome. Gynecol Obstet Fertil 2005;33:7504. [Article in French].

5. Souka AP, Pilalis A, Kavalakis I, Antsaklis P, Papantoniou N, Mesogitis S, Antsaklis A. Screening for major structural abnormalities at the 11-14 weeks scan. Am J Obstet Gynecol 2006;194:393-6.

6. Sanseverino MT, de Souza CF, Gissen P, Sordi AO,
Magalhaes JA, Schuler-Faccini L. Increased nuchal translucency in arthrogriposis, renal dysfunction and cholestasis syndrome and discovery of a Portuguese specific mutation in VPS33B gene. Ultrasound Obstet Gynecol 2006;28:233-4.

7. Allem S, Howarth ES. Apert syndrome associated with increased fetal nuchal translucency. Prenat Diagn 2005;25:1066-7.

8. Souka AP, Skentou H, Greets L, Bower S, Nicolaides $\mathrm{KH}$. Congenital nephritic syndrome presenting with increased nuchal translucency in the first trimester. Prenat Diagn 2002;22:93-5.

9. Atzei A, Gajewska K, Huggon IC, Allan L, Nicolaides $\mathrm{KH}$. Relationship between nuchal translucency thickness and prevalence of major cardiac defects with normal kariotype. Ultrasound Obstet Gynecol 2005;26:154-7. 